\title{
Erratum to: Evolution Law of the Optical Field of Degenerate Parametric Amplifier in Dissipative Channel
}

\author{
Feng Chen · Bao-long Fang $\cdot$ Rui He $\cdot$ Hong-yi Fan
}

Published online: 30 August 2014

(C) Springer Science+Business Media New York 2014

Erratum to: Int J Theor Phys (2014) 53:2846-2854

DOI 10.1007/s10773-014-2082-0

There was an error in the funding acknowledgement of the paper referenced above. The corrected acknowledgement reads "F. Chen work was supported by the General Project of the Research and Development Foundation of Hefei University (14KY14ZR), and the National Natural Science Foundation of China under grant 11247009.”

The online version of the original article can be found at http://doi:10.1007/s10773-014-2082-0.

F. Chen $(\bowtie) \cdot$ B.-1.Fang $\cdot$ H.-y. Fan

Department of Mathematics and Physics, Hefei University, Hefei, Anhui 230601, China e-mail: chenfeng@hfuu.edu.cn

F. Chen $\cdot$ R. He $\cdot$ H.-y. Fan

Department of Material Science and Engineering, University of Science and Technology of China, Hefei, Anhui 230026, People's Republic of China 